\title{
$K$-theoretic matching of brane charges in S- and U-duality
}

\author{
Stefan Mendez-Diez ${ }^{1}$ and Jonathan Rosenberg ${ }^{2}$ \\ ${ }^{1}$ Department of Mathematical and Statistical Sciences, \\ University of Alberta, \\ Edmonton, AB T6G 2G1, Canada \\ mendezdi@ualberta.ca \\ ${ }^{2}$ Department of Mathematics, \\ University of Maryland, \\ College Park, MD 20742-4015, USA \\ jmr@math.umd.edu
}

\begin{abstract}
We discuss $K$-theoretic matching of $D$-brane charges in the string duality between type I on $\mathbb{T}^{4}$ and type IIA on $K 3$. This case is more complex than the familiar case of IIA/IIB duality, which is already well understood, but it turns out that replacing $K 3$ by its orbifold blow-down seems to largely resolve the apparent problems with the theory.
\end{abstract}

\section{Introduction}

It is believed that the five superstring theories are all related through various dualities: T-duality, S-duality, and a combination of both, known as U-duality [17]. Sometimes the explicit dualities between the different superstring theories are unclear.

e-print archive: http://lanl.arXiv.org/abs/1007.1202v3 
"ATMP-16-6-A1-MEN" — 2013/5/22 — 21:05 — page 1592 — \#2

We can determine a lot about possible dualities by looking at stable $D$-brane configurations. If two theories are dual to one another then they should have the same spectra. Therefore, dual string theories should have equivalent brane configurations. (Equivalent here means, for instance, that it should be possible to match up the brane charges in the two theories, and thus these charges should live in isomorphic groups.) The stable $D$-brane configurations in a given theory depend only on the topology of the spacetime and can be classified by [possibly twisted] $K$-theory $[24,25,37,38]$. The $K$-theoretic classification of $D$-brane charges has proven very useful in the study of string dualities, particularly $T$-duality between the type IIA and IIB theories. By putting together the known duality between type I and the $\mathrm{SO}(32)$ heterotic string theories with the one between the $\mathrm{SO}(32)$ heterotic and type IIA string theories, one obtains an example of a U-duality between type I and IIA string theories. This paper will focus on matching the $K$-theoretic classification of $D$-brane charges in this example of $U$-duality.

It is conjectured that type IIA string theory on $K 3$ is dual to the $\mathrm{SO}(32)$ heterotic string on the 4-torus, $\mathbb{T}^{4}[16]$, [36, Section 4], [4,30,34], [3, p. 424], $[1,19]$. The $\mathrm{SO}(32)$ heterotic string is believed to be equivalent to type I string theory via S-duality $[8,15,35]$, so this gives a duality between type I string theory on $\mathbb{T}^{4}$ and type IIA string theory on $K 3$. (This chain of equivalences is mentioned explicitly in [29, p. 258].) Not only does this give a concrete example of a duality between types I and IIA theories, but it can be extended to relate all five of the superstring theories. At the $\mathbb{T}^{4} / \mathbb{Z}_{2}$ point of the moduli space, the type IIA theory can be related by a $T$-duality to the type IIB theory on $\mathbb{T}^{4} / \mathbb{Z}_{2}$ with an $N S 5$-brane at each of the 16 singularities $[1,20,31]$. This is also related to the type I theory on $\mathbb{T}^{4}$ via $S$-duality [31]. Furthermore the $\mathrm{SO}(32)$ and $E_{8} \times E_{8}$ heterotic theories compactified on a torus are $T$-dual to one another [3, p. 288].

Stable $D$-brane charges are classified by $K O(X)$ in type I string theory $[26,37]$, and by $\widetilde{K}(X)$ (resp., $K^{-1}(X)$ ) in type IIB theory (resp., type IIA theory) $[3,37,38]$. Since it is conjectured that type I string theory on $\mathbb{T}^{4}$ and type IIA string theory on $K 3$ are dual to each other, they should have the same possible charges. The puzzle is that $K O^{*}\left(\mathbb{T}^{4}\right)$ contains 2-torsion (implying the existence of torsion charged $D$-branes in the type I theory compactified on $\left.\mathbb{T}^{4}\right)$ and $K^{0}(K 3) \cong \mathbb{Z}^{24}$, while $K^{-1}(K 3) \cong 0$, so that there wouldn't appear to be any stable $D$-brane charges at all in type IIA theory compactified on $K 3$ ! Even in IIB theory on $K 3$, it appears there is no room for torsion brane charges!

Now one could object that $D$-branes in one theory do not necessarily transform to $D$-branes in a dual theory. However, if there is a $D$-brane in one theory, there must be an object (not necessarily a $D$-brane) in the 
dual theory that corresponds to the $D$-brane with the correct charge. For example, under the duality between the type IIA theory on $K 3$ and the $\mathrm{SO}(32)$ heterotic theory on $\mathbb{T}^{4}$, the fundamental heterotic string appears as a non-singular soliton solution in the type IIA theory. Similarly, the fundamental type IIA string appears as a non-singular soliton solution in the heterotic theory [30]. The spectrum of the elementary heterotic string contains charged Bogomol'nyi-Prasad-Sommerfield (BPS) states, but the spectrum of the elementary type IIA string does not. All of the gauge fields in the type IIA theory come from the Ramond-Ramond (RR) sector, so the only charged BPS states are $D$-branes. The elementary type IIA string is not charged. This is explained by the fact that the charged states in the type IIA theory appear as solitons in the heterotic theory and not in the elementary string spectrum. The fundamental type IIA string appears as an uncharged soliton in the heterotic theory. Even though $D$-branes do not necessarily transform to $D$-branes, it is possible to match the charges in the dual theories. In our current example, there is no way to explain the torsion charges that appear in the type I theory in the type IIA theory other than as torsion charged $D$-branes. The NS5-brane that wraps the $K 3$ appearing in the type IIA theory corresponds to the fundamental string in the heterotic theory. Since the fundamental heterotic string is BPS, it cannot have torsion charge. Therefore, the NS5-brane occurring in the type IIA theory cannot have torsion charge and the torsion charged branes occurring in the type I theory must map to torsion charged $D$-branes in the type IIA theory. There must be $D$-branes in the type IIA theory that carry torsion charge, but there is no torsion in the cohomology or [untwisted] $K$-theory of $K 3$. Furthermore, twisting by an $H$-flux cannot introduce torsion in the twisted $K$-theory of $K 3$ since any twist would have to live in $H^{3}(K 3)=0$. We show that if one first removes the sixteen isolated singularity points of an orbifold blow-down of $K 3$, such an isomorphism is close to being achieved, albeit in a very non-trivial way. Thus this calculation provides an interesting test of $\mathrm{S}$ - and U-duality. We then look at the K-theory classification of $D$-brane charges at the orbifold point, $\mathbb{T}^{4} / \mathbb{Z}_{2}$, of the moduli space of $K 3$ for both the type IIA and IIB theories and discuss some of the issues that arise.

The first author would like to thank Chuck Doran for many useful conversations and suggestions.

\section{$2 K O^{*}\left(\mathbb{T}^{4}\right)$}

We work throughout with $K$-theory with compact support. Thus for a locally compact space $X$, which is not compact, $K O^{*}(X)$ is (essentially by definition) identified with $\widetilde{K O}^{*}\left(X^{+}\right)$, where $X^{+}=X \cup\{\infty\}$ is the one-point 
compactification of $X$ (e.g., $\left.\left(\mathbb{R}^{n}\right)^{+}=S^{n}\right) . K O^{-n}\left(\mathbb{T}^{4}\right)$ can be computed from

$$
K O^{-i}(\mathrm{pt}) \cong \begin{cases}\mathbb{Z}, & i \equiv 0 \quad(\bmod 4) \\ \mathbb{Z}_{2}, & i \equiv 1,2(\bmod 8), \\ 0, & \text { otherwise },\end{cases}
$$

by iterating the formula $K O^{k}\left(X \times S^{1}\right) \cong K O^{k}(X) \oplus K O^{k-1}(X)$, which follows from the axioms of a (generalized) cohomology theory. Thus, we obtain:

$$
\begin{aligned}
K O^{-i}\left(\mathbb{T}^{4}\right) \cong & K O^{-i}\left(\mathbb{T}^{3}\right) \oplus K O^{-(i+1)}\left(\mathbb{T}^{3}\right) \\
\cong & K O^{-i}\left(\mathbb{T}^{2}\right) \oplus 2 K O^{-(i+1)}\left(\mathbb{T}^{2}\right) \oplus K O^{-(i+2)}\left(\mathbb{T}^{2}\right) \\
\cong & K O^{-i}(\mathbb{T}) \oplus 3 K O^{-(i+1)}(\mathbb{T}) \oplus 3 K O^{-(i+2)}(\mathbb{T}) \oplus K O^{-(i+3)}(\mathbb{T}) \\
\cong & K O^{-i}(\mathrm{pt}) \oplus 4 K O^{-(i+1)}(\mathrm{pt}) \oplus 6 K O^{-(i+2)}(\mathrm{pt}) \\
& \oplus 4 K O^{-(i+3)}(\mathrm{pt}) \oplus K O^{-(i+4)}(\mathrm{pt}) .
\end{aligned}
$$

Since type I string theory is a ten-dimensional theory, the actual spacetime manifold for type I string theory compactified on $\mathbb{T}^{4}$ is $\mathbb{T}^{4} \times \mathbb{R}^{6}$. Stable $D$-brane charges in type I string theory on $\mathbb{T}^{4} \times \mathbb{R}^{6}$ are thus classified by

$$
K O^{0}\left(\mathbb{T}^{4} \times \mathbb{R}^{6}\right) \cong K O^{-6}\left(\mathbb{T}^{4}\right) \cong 6 \mathbb{Z} \oplus 5 \mathbb{Z}_{2} .
$$

However, this may not be the end of the story. If $\iota: Y^{p+1} \hookrightarrow \mathbb{T}^{4} \times \mathbb{R}^{6}$ is the inclusion of a (proper) $\mathrm{D} p$-brane in $\mathbb{T}^{4} \times \mathbb{R}^{6}$, with $Y$ assumed to be spin for anomaly cancellation, the Gysin map in $K O$-theory gives a map ı! $K O(Y) \rightarrow K O^{3-p}\left(\mathbb{T}^{4}\right)$ obtained as the following composite:

$$
\begin{aligned}
K O(Y) & \stackrel{\text { P.D. }}{\longrightarrow} K O_{p+1}(Y) \stackrel{\iota_{*}}{\longrightarrow} K O_{p+1}\left(\mathbb{T}^{4} \times \mathbb{R}^{6}\right) \\
& \stackrel{\text { (P.D. })^{-1}}{\longrightarrow} K O^{10-(p+1)}\left(\mathbb{T}^{4} \times \mathbb{R}^{6}\right) \cong K O^{3-p}\left(\mathbb{T}^{4}\right) .
\end{aligned}
$$

Here P.D. denotes the Poincaré duality isomorphism and (P.D. $)^{-1}$ is its inverse. A Chan-Paton bundle with orthogonal gauge group gives a class in $K O(Y)$, and thus via the Gysin map $\iota$ ! a $D$-brane charge in $K O^{3-p}\left(\mathbb{T}^{4}\right)$. This is $K O^{-6}\left(\mathbb{T}^{4}\right) \cong 6 \mathbb{Z} \oplus 5 \mathbb{Z}_{2}$ when $p=9$ or 1 . (Recall that real $K$-theory satisfies Bott periodicity with period 8.) Similarly, a Chan-Paton bundle with symplectic gauge group gives a class in $K S p(Y) \cong K O^{4}(Y)$ (since real and symplectic $K$-theory agree after a dimension shift by 4 ), and thus via the Gysin map $\iota$ ! a $D$-brane charge in $K O^{7-p}\left(\mathbb{T}^{4}\right)$. This can again be identified with $K O^{-6}\left(\mathbb{T}^{4}\right)$ when $p=5$. The 9-branes and 1-branes with real Chan-Paton bundles, along with the 5-branes with symplectic ChanPaton bundles, account for all the usual BPS-branes of type I superstring 
Table 1: Groups of D p-brane charges for type I compactified on $\mathbb{T}^{4}$

\begin{tabular}{|c|c|c|c|}
\hline$p$ & Bundle type & BPS? & Charge group \\
\hline 9 & $\mathrm{O}$ & Yes & $K O^{-6}\left(\mathbb{T}^{4}\right) \cong 6 \mathbb{Z} \oplus 5 \mathbb{Z}_{2}$ \\
\hline 9 & $\mathrm{Sp}$ & No & $K O^{-2}\left(\mathbb{T}^{4}\right) \cong 6 \mathbb{Z} \oplus \mathbb{Z}_{2}$ \\
\hline 8 & $\mathrm{O}$ & No & $K O^{-5}\left(\mathbb{T}^{4}\right) \cong 4 \mathbb{Z} \oplus \mathbb{Z}_{2}$ \\
\hline 8 & $\mathrm{Sp}$ & No & $K O^{-1}\left(\mathbb{T}^{4}\right) \cong 4 \mathbb{Z} \oplus 5 \mathbb{Z}_{2}$ \\
\hline 7 & $\mathrm{O}$ & No & $K O^{-4}\left(\mathbb{T}^{4}\right) \cong 2 \mathbb{Z}$ \\
\hline 7 & $\mathrm{Sp}$ & No & $K O^{0}\left(\mathbb{T}^{4}\right) \cong 2 \mathbb{Z} \oplus 10 \mathbb{Z}_{2}$ \\
\hline 6 & $\mathrm{O}$ & No & $K O^{-3}\left(\mathbb{T}^{4}\right) \cong 4 \mathbb{Z}$ \\
\hline 6 & $\mathrm{Sp}$ & No & $K O^{1}\left(\mathbb{T}^{4}\right) \cong 4 \mathbb{Z} \oplus 10 \mathbb{Z}_{2}$ \\
\hline 5 & $\mathrm{O}$ & No & $K O^{-2}\left(\mathbb{T}^{4}\right) \cong 6 \mathbb{Z} \oplus \mathbb{Z}_{2}$ \\
\hline 5 & $\mathrm{Sp}$ & Yes & $K O^{2}\left(\mathbb{T}^{4}\right) \cong 6 \mathbb{Z} \oplus 5 \mathbb{Z}_{2}$ \\
\hline 4 & $\mathrm{O}$ & No & $K O^{-1}\left(\mathbb{T}^{4}\right) \cong 4 \mathbb{Z} \oplus 5 \mathbb{Z}_{2}$ \\
\hline 4 & Sp & No & $K O^{3}\left(\mathbb{T}^{4}\right) \cong 4 \mathbb{Z} \oplus \mathbb{Z}_{2}$ \\
\hline 3 & $\mathrm{O}$ & No & $K O^{0}\left(\mathbb{T}^{4}\right) \cong 2 \mathbb{Z} \oplus 10 \mathbb{Z}_{2}$ \\
\hline 3 & $\mathrm{Sp}$ & No & $K O^{4}\left(\mathbb{T}^{4}\right) \cong 2 \mathbb{Z}$ \\
\hline 2 & $\mathrm{O}$ & No & $K O^{1}\left(\mathbb{T}^{4}\right) \cong 4 \mathbb{Z} \oplus 10 \mathbb{Z}_{2}$ \\
\hline 2 & Sp & No & $K O^{5}\left(\mathbb{T}^{4}\right) \cong 4 \mathbb{Z}$ \\
\hline 1 & $\mathrm{O}$ & Yes & $K O^{2}\left(\mathbb{T}^{4}\right) \cong 6 \mathbb{Z} \oplus 5 \mathbb{Z}_{2}$ \\
\hline 1 & $\mathrm{Sp}$ & No & $K O^{6}\left(\mathbb{T}^{4}\right) \cong 6 \mathbb{Z} \oplus \mathbb{Z}_{2}$ \\
\hline 0 & $\mathrm{O}$ & No & $K O^{3}\left(\mathbb{T}^{4}\right) \cong 4 \mathbb{Z} \oplus \mathbb{Z}_{2}$ \\
\hline 0 & Sp & No & $K O^{7}\left(\mathbb{T}^{4}\right) \cong 4 \mathbb{Z} \oplus 5 \mathbb{Z}_{2}$ \\
\hline-1 & $\mathrm{O}$ & No & $K O^{4}\left(\mathbb{T}^{4}\right) \cong 2 \mathbb{Z}$ \\
\hline-1 & $\mathrm{Sp}$ & No & $K O^{8}\left(\mathbb{T}^{4}\right) \cong 2 \mathbb{Z} \oplus 10 \mathbb{Z}_{2}$ \\
\hline
\end{tabular}

theory [3, p. 223]. However, as pointed out by many authors, e.g., [2, 5, $6,11,13,32,33,37$, there can be additional $D$-brane charges coming from non-supersymmetric, but still stable, branes with other values of $p$. Such charges (for type-I superstring theory compactified on $\mathbb{T}^{4}$ ) are summarized in Table 1. The various kinds of branes are hypothetical; not all of them actually occur. Also note that after inverting $2, K O$ and $K S p$ are the same, so the nature of the Chan-Paton gauge group only affects the 2-torsion.

\section{$3 \quad K$-Theory of a desingularized $K 3$}

It becomes immediately obvious that we do not want to just use $K 3$ as a possible dual topology to type I theory on $\mathbb{T}^{4}$, because the complex $K$-theory of $K 3$ contains no torsion, and $K^{-1}(K 3) \cong 0$, which would imply there 
"ATMP-16-6-A1-MEN" — 2013/5/22 — 21:05 — page 1596 — \#6

were no stable $D$-branes in the type IIA theory compactified on $K 3$ and we could not explain the torsion charges occurring in the type I theory. Instead of $K 3$, a $\mathbb{Z}_{2}$ orbifold quotient of $\mathbb{T}^{4}$, where $\mathbb{Z}_{2}$ acts by multiplication by -1 (which is a singular limit of $K 3$ ), is often used in string theory because the Ricci-flat metric can be explicitly determined [3, Section 9.3]. The orbifold, $\mathbb{T}^{4} / \mathbb{Z}_{2}$ has 16 isolated singular points, the 16 fixed points of the $\mathbb{Z}_{2}$ action on $\mathbb{T}^{4}$. We can remove these 16 singular points by first removing 16 open balls in $\mathbb{T}^{4}$ surrounding each of the singular points. We then divide out by the $\mathbb{Z}_{2}$ action on $\mathbb{T}^{4}$ minus the 16 open balls to obtain a smooth manifold (with boundary), $N$. The boundary of $N$ is 16 copies of $\mathbb{R P}^{3}$. 16 copies of an Eguchi-Hanson space (which topologically is the unit disk bundle of the tangent bundle of $S^{2}=\mathbb{C P}^{1}$ ) are usually glued onto $N$ along their common boundary to create a manifold with the same topology as $K 3$. This cannot be the correct manifold for our purposes because again its cohomology (and thus its $K$-theory) contains no torsion. Since the original $K 3$ in the type IIA theory has no singularities, we do not want to allow for any physical effect from the singularities (which like $D$-branes are sources for RR charges). Therefore, physically, we are only interested in fields that approach a constant value at the singularities, and it makes more sense simply to collapse the singularities and deal with the singular quotient space $\left(\mathbb{T}^{4} / \mathbb{Z}_{2}\right) /($ singularities $) \cong N / \partial N$. Since this space is the one-point compactification of the interior of $N$, we are interested in $\widetilde{K}^{*}(N / \partial N) \cong K^{*}(N, \partial N)$, the relative $K$-theory of the manifold $N$ rel its boundary. Although we do not want to allow for any effect from the singularities in the type IIA theory, the situation is different when looking at the type IIB theory.

The type IIA theory compactified on $\mathbb{T}^{4} / \mathbb{Z}_{2}$, the orientifold point of the moduli space of $K 3$, is $T$-dual to the type IIB theory compactified on $\mathbb{T}^{4} / \mathbb{Z}_{2}$ with an NS5-brane and an orientifold 5-plane at each of the 16 fixed points of the $\mathbb{Z}_{2}$ action. Performing an $\mathrm{SL}(2, \mathbb{Z})$ transformation on this configuration transforms the $N S 5$-branes to $D 5$-branes, giving us the type IIB theory on $\mathbb{T}^{4} / \mathbb{Z}_{2}$ with a $D 5$-brane and an $O 5$-plane at each of the singularities $[1,20,31]$. The final type IIB configuration is equivalent to the type I theory on $\mathbb{T}^{4}$. This gives us an explicit way of relating type I on $\mathbb{T}^{4}$, type IIA on $K 3$, and type IIB on $\mathbb{T}^{4} / \mathbb{Z}_{2}$ with either an $N S 5$-brane or a $D 5$-brane at each of the $O 5$-planes. The $T$-duality transformation between the type IIA theory and the type IIB theory transforms the 64 blow-up modes of the type IIA theory at the orbifold point of the moduli space (4 for each singularity) into moduli controlling the positions of the 5 -branes. While we described this duality for the case when the 5-branes are located at the singularities, the duality is true when the branes are moved [1]. With this in mind, we will move the 5-branes a small distance from the singularities and consider them located on the boundary of $N$. In this case, the effects of the boundary are 
important and we cannot mod them out. Therefore, in the type IIB theory we are interested in $K^{*}(N)$.

To compute $K^{*}(N, \partial N)$ and $K^{*}(N)$ we will first need to compute the homology of $N$. Let $M$ be $\mathbb{T}^{4} \backslash$ (16 open balls), which is the double cover of $N$. Since $N$ is obtained from $M$ by dividing out by a free $\mathbb{Z}_{2}$-action, there is a spectral sequence $H_{p}\left(\mathbb{Z}_{2}, H_{q}(M)\right) \Rightarrow H_{p+q}(N)$. (See for example [23, Theorem $\left.8^{\text {bis }} .9\right]$.) So, we must first determine the homology of $M$ as a $\mathbb{Z}_{2}$-module. The homology of $M$ is torsion-free as a $\mathbb{Z}$-module, but we need to compute it as a $\mathbb{Z} G$-module, where $G=\mathbb{Z}_{2}$. Since $G$ is self-dual, the ring $\mathbb{Z} G$ is isomorphic to the representation $\operatorname{ring} R$ of $G$ studied in the Appendix (Section A), so we refer to results there for more details about homological algebra over this ring. In particular, it turns out (as a consequence of iterated application of Propositions A.2, A.4, A.5, and A.6) that the homology of $M$ is a direct sum of copies of three standard $R$-modules (all torsion-free as $\mathbb{Z}$-modules): $R$ as a module over itself, the trivial module $\mathbb{Z}$ (or $R / I$, in the notation of the Appendix), and $\mathbb{Z}$ with the non-trivial $\mathbb{Z}_{2}$-action (where the generator of the group acts by multiplication by -1 ), which we call $\underline{\mathbb{Z}}$ to distinguish it from $\mathbb{Z}$ with the trivial $\mathbb{Z}_{2}$-action. (This last $R$-module, in the notation of the Appendix, is $R / J$.)

First of all, note that the cohomology ring of $\mathbb{T}^{4}$ is an exterior algebra on 4 generators. Each of these generators is sent to its negative under the $\mathbb{Z}_{2}$ action, so $\mathbb{Z}_{2}$ acts trivially on the even exterior powers and non-trivially on the odd exterior powers. So $H^{1}\left(\mathbb{T}^{4}\right) \cong H_{1}\left(\mathbb{T}^{4}\right) \cong H^{3}\left(\mathbb{T}^{4}\right) \cong H_{3}\left(\mathbb{T}^{4}\right) \cong \underline{\mathbb{Z}}^{4}$, whereas $H^{2}\left(\mathbb{T}^{4}\right) \cong H_{2}\left(\mathbb{T}^{4}\right) \cong \mathbb{Z}^{6}$. Now by a simple transversality argument, removing 16 balls from $\mathbb{T}^{4}$ does not change the fundamental group, so $\pi_{1}(M) \cong \pi_{1}\left(\mathbb{T}^{4}\right) \cong \underline{\mathbb{Z}}^{4}$. Therefore, $H_{1}(M) \cong \underline{\mathbb{Z}}^{4}$. To obtain $H_{2}(M)$ we can use the Meyer-Vietoris sequence:

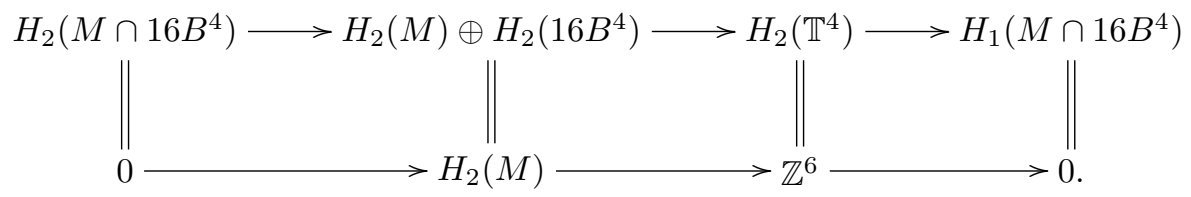

Here $B^{4}$ is the closed 4-ball, so $M \cap 16 B^{4}=16 S^{3}$. So we see $H_{2}(M) \cong \mathbb{Z}^{6}$. We can determine $H_{3}(M)$ from the long exact sequence of pairs, using the pair $(M, \partial M)$, where $\partial M=16 S^{3}$. The part of the long exact sequence we are interested in is

$H_{4}(M) \rightarrow H_{4}(M, \partial M) \rightarrow H_{3}\left(16 S^{3}\right) \rightarrow H_{3}(M) \rightarrow H_{3}(M, \partial M) \rightarrow H_{2}\left(16 S^{3}\right)$.

$H_{4}(M) \cong 0$ since $M$ has a nonempty boundary. By Poincaré duality $H_{4}(M, \partial M) \cong H^{0}(M) \cong H_{0}(M) \cong \mathbb{Z}$. Similarly, $H_{3}(M, \partial M) \cong H^{1}(M) \cong$ 
$F H_{1}(M) \oplus T H_{0}(M) \cong \underline{\mathbb{Z}}^{4}$. Finally, $H_{3}\left(16 S^{3}\right) \cong \mathbb{Z}^{16}$ (the $\mathbb{Z}_{2}$ action is trivial since it preserves orientation on $\left.S^{3}\right)$ and $H_{2}\left(16 S^{3}\right) \cong 0$. Putting this all together, the long exact sequence becomes

$$
0 \rightarrow \mathbb{Z} \rightarrow \mathbb{Z}^{16} \rightarrow H_{3}(M) \rightarrow \underline{\mathbb{Z}}^{4} \rightarrow 0
$$

where it is easy to see that the map $\mathbb{Z} \rightarrow \mathbb{Z}^{16}$ is just the diagonal inclusion. This shows us that $H_{3}(M)$ is an extension of $\underline{\mathbb{Z}}^{4}$ by $\mathbb{Z}^{15}$. To summarize, we have

$$
H_{i}(M) \cong \begin{cases}\mathbb{Z}, & i=0, \\ \underline{\mathbb{Z}}^{4}, & i=1, \\ \mathbb{Z}^{6}, & i=2, \\ \text { extension of } \underline{\mathbb{Z}}^{4} \text { by } \mathbb{Z}^{15}, & i=3, \\ 0, & \text { otherwise. }\end{cases}
$$

However, it will turn out that the extension in $H_{3}(M)$ cannot be split, and rather is $R^{4} \oplus \mathbb{Z}^{11}$. To see this, observe that the spectral sequence $E_{p, q}^{2}=H_{p}\left(\mathbb{Z}_{2}, H_{q}(M)\right) \Rightarrow H_{p+q}(N)$ has only four rows, and thus $E^{5}=E^{\infty}$. Recall that $H_{p}\left(\mathbb{Z}_{2}, \mathbb{Z}\right) \cong \mathbb{Z}$ for $p=0, \mathbb{Z}_{2}$ for $p \geq 1$ odd, and 0 for $p \geq 2$ even, while $H_{p}\left(\mathbb{Z}_{2}, \underline{\mathbb{Z}}\right) \cong \mathbb{Z}_{2}$ for $p \geq 0$ even, 0 for $p$ odd, so the first 3 rows of $E^{2}$ are:

Table 2: $E^{2}$ of the spectral sequence for computing $H_{*}(N)$

$$
\begin{array}{l|lllllll}
2 & \mathbb{Z}^{6} & \mathbb{Z}_{2}^{6} & 0 & \mathbb{Z}_{2}^{6} & 0 & \mathbb{Z}_{2}^{6} & \cdots \\
1 & \mathbb{Z}_{2}^{4} & 0 & \mathbb{Z}_{2}^{4} & 0 & \mathbb{Z}_{2}^{4} & 0 & \cdots \\
0 & \mathbb{Z} & \mathbb{Z}_{2} & 0 & \mathbb{Z}_{2} & 0 & \mathbb{Z}_{2} & \cdots \\
\hline q / p & 0 & 1 & 2 & 3 & 4 & 5 & \cdots
\end{array}
$$

Since $N$ is a noncompact 4-manifold, its homology must vanish in dimension 4 and higher, so $E_{p, 3}^{2}$ for $p \geq 1$ must be killed off by entries in the rows with $q \leq 2$ and total degree $p+4 \geq 5$. If $H_{3}(M)$ were to contain a summand isomorphic to $\underline{\mathbb{Z}}$, then $E_{p, 3}^{2}$ would contain 2 -torsion for all even $p$, which contradicts the fact that $E_{p, q}^{2}=0$ for $q \leq 2, p>0$, and $p+q$ even. Thus, by iterated application of Propositions A.2, A.4, A.5, and A.6, $H_{3}(M) \cong \mathbb{Z}^{11} \oplus(\mathbb{Z} G)^{4}$. It follows that the $q=3$ row of $E^{2}$ in the spectral sequence is $\mathbb{Z}^{15}$ for $p=0, \mathbb{Z}_{2}^{11}$ for $p \geq 1$ odd, and 0 otherwise.

We can now determine $H_{*}(N)$ from the spectral sequence with $E_{p, q}^{2}=$ $H_{p}\left(\mathbb{Z}_{2}, H_{q}(M)\right.$ ) (Table 2). For $p>0, E_{p, q}^{2}$ is all torsion, so the free part of 
$H_{q}(N)$ is the same as for $E_{0, q}^{2}$. So we see that the Betti numbers of $N$ are

$$
\beta_{i}(N)= \begin{cases}1, & i=0 \\ 0, & i=1 \\ 6, & i=2 \\ 15, & i=3 \\ 0, & \text { otherwise }\end{cases}
$$

First we know that $H_{0}(N) \cong \mathbb{Z}$ since $N$ is connected. We also know $H_{4}(N) \cong$ 0 because $N$ has a nonempty boundary. Now $H_{3}(N) \cong H^{1}(N, \partial N) \cong$ $F H_{1}(N, \partial N) \oplus T H_{0}(N, \partial N)$, and $T H_{0}(N, \partial N) \cong 0$. So, $H_{3}(N)$ is free, and thus isomorphic to $\mathbb{Z}^{15}$. We can use Mayer-Vietoris with $N$ and 16 EguchiHanson spaces, $E$, since $N \cup_{16 \mathbb{R}^{3}} 16 E \cong K 3$, to show that $H_{2}(N) \cong \mathbb{Z}^{6}$, from the exact sequence

$$
\cdots \rightarrow H_{k+1}(K 3) \rightarrow H_{k}\left(16 \mathbb{R} \mathbb{P}^{3}\right) \rightarrow H_{k}(N) \oplus H_{k}(16 E) \rightarrow H_{k}(K 3) \rightarrow \cdots
$$

Furthermore, $E$ has the same homotopy type as $S^{2}$, since it is the unit disk bundle of the tangent bundle of $S^{2}$. The part of the Mayer-Vietoris sequence we are interested in is:

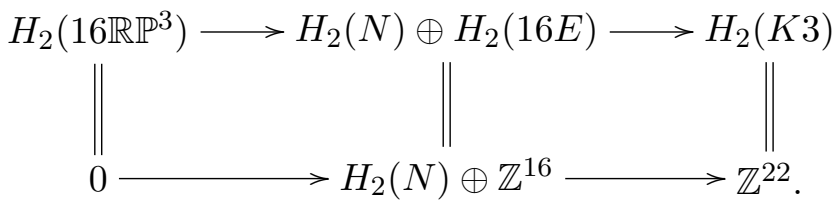

From this, we see that $H_{2}(N)$ injects into a free abelian group and thus must be free. We have shown that only $H_{1}(N)$ can have any torsion.

We can calculate $H_{1}(N)$ from the spectral sequence $H_{*}\left(\mathbb{Z}_{2}, H_{*}(M)\right) \Rightarrow$ $H_{*}(N)$.

$$
E_{1,0}^{2}=H_{1}\left(\mathbb{Z}_{2}, H_{0}(M)\right) \cong \mathbb{Z}_{2}
$$

No non-zero differential hits it or leaves it because we have a first quadrant spectral sequence.

$$
E_{0,1}^{2}=H_{0}\left(\mathbb{Z}_{2}, H_{1}(M)\right) \cong \mathbb{Z}_{2}^{4}
$$

Again no differential hits it since $E_{2,0}^{2}=H_{2}\left(\mathbb{Z}_{2}, \mathbb{Z}\right) \cong 0$. Therefore, $H_{1}(N)$ is an extension of $\mathbb{Z}_{2}$ by $\mathbb{Z}_{2}^{4}$. Also $H_{1}(N)$ is a quotient of $\mathbb{Z}_{2}{ }^{16}$ as can be 
seen from Mayer-Vietoris:

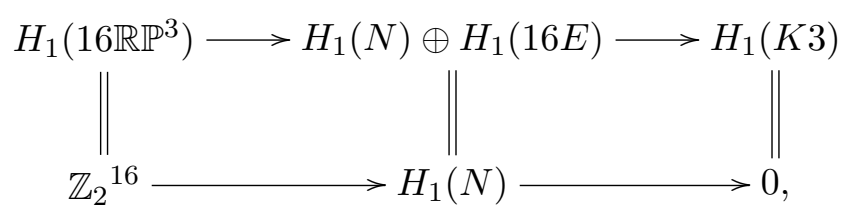

so all of its torsion is of order 2. Therefore, the extension is trivial and $H_{1}(N) \cong \mathbb{Z}_{2}^{5}$. Putting this all together, we see that

$$
H_{i}(N) \cong \begin{cases}\mathbb{Z}, & i=0, \\ \mathbb{Z}_{2}^{5}, & i=1, \\ \mathbb{Z}^{6}, & i=2, \\ \mathbb{Z}^{15}, & i=3, \\ 0, & \text { otherwise. }\end{cases}
$$

By Poincaré duality for manifolds with boundary (also known as AlexanderLefschetz duality), the cohomology of $N$ relative to its boundary is

$$
H^{i}(N, \partial N) \cong H_{4-i}(N) \cong \begin{cases}0, & i=0, \\ \mathbb{Z}^{15}, & i=1, \\ \mathbb{Z}^{6}, & i=2, \\ \mathbb{Z}_{2}^{5}, & i=3, \\ \mathbb{Z}, & i=4, \\ 0, & \text { otherwise }\end{cases}
$$

The $K$-theory is then computed from the Atiyah-Hirzebruch spectral sequence

$$
H^{p}\left(N, K^{q}(\mathrm{pt})\right) \Rightarrow K^{p+q}(N),
$$

but all differentials vanish since the first differential is the Steenrod operation $\mathrm{Sq}^{3}$, which must vanish, and there is no room in this case for any higher differentials. Since the spectral sequence collapses at $E_{2}$ and $\widetilde{H}^{2}(N) \cong \mathbb{Z}^{6} \oplus$ $\mathbb{Z}_{2}^{5}$, while $H^{1}(N)=0$ and $H^{3}(N) \cong \mathbb{Z}^{15}$,

$$
\left\{\begin{array}{l}
K^{0}(N) \cong \mathbb{Z}^{7} \oplus \mathbb{Z}_{2}^{5}, \\
K^{-1}(N) \cong \mathbb{Z}^{15}
\end{array}\right.
$$

The Universal Coefficient Theorem in $K$-theory (see [21] for references) gives a short exact sequence

$$
0 \rightarrow \operatorname{Ext}_{\mathbb{Z}}^{1}\left(K^{k+1}(N), \mathbb{Z}\right) \rightarrow K_{k}(N) \rightarrow \operatorname{Hom}\left(K^{k}(N), \mathbb{Z}\right) \rightarrow 0,
$$


that splits (non-canonically), so $K_{0}(N) \cong \mathbb{Z}^{7}$ and

$$
K_{1}(N) \cong \mathbb{Z}^{15} \oplus \operatorname{Ext}_{\mathbb{Z}}^{1}\left(\mathbb{Z}^{7} \oplus \mathbb{Z}_{2}^{5}, \mathbb{Z}\right) \cong \mathbb{Z}^{15} \oplus \mathbb{Z}_{2}^{5}
$$

Finally, since $N$ is an even-dimensional compact $\operatorname{spin}^{c}$ manifold (with boundary), we have Poincaré duality

$$
K_{*}(N) \cong K^{*}(N, \partial N) .
$$

So $K^{0}(N, \partial N) \cong K_{0}(N) \cong \mathbb{Z}^{7}$ and $K^{-1}(N, \partial N) \cong K_{1}(N) \cong \mathbb{Z}^{15} \oplus \mathbb{Z}_{2}{ }^{5}$.

The stable $D$-brane charges in the type IIB theory on $N \times \mathbb{R}^{6}$ with 16 5 -branes on its boundary are classified by $K^{0}\left(N \times \mathbb{R}^{6}\right) \cong K^{0}(N) \cong \mathbb{Z}^{7} \oplus \mathbb{Z}_{2}^{5}$. In the type IIA theory, the stable D-brane charges on $\stackrel{N}{ } \times \mathbb{R}^{6}$ are classified by $K^{-1}\left((N / \partial N) \times \mathbb{R}^{6}\right) \cong K^{-1}(N / \partial N) \cong K^{-1}(N, \partial N) \oplus K^{-1}(\mathrm{pt}) \cong \mathbb{Z}^{15} \oplus$ $\mathbb{Z}_{2}^{5}\left(\right.$ since $\left.K^{-1}(\mathrm{pt}) \cong 0\right)$.

\section{$4 D$-Brane charges in $\mathbb{Z}_{2}$-Equivariant $K$-Theory}

Since $N$ was obtained from the orbifold $\mathbb{T}^{4} / \mathbb{Z}_{2}$, we can obtain more information about the classification of D-branes on $(N, \partial N)$ by first looking at how D-branes are classified on $\mathbb{T}^{4} / \mathbb{Z}_{2}$.

As described in [37], [18], [12] and [14], stable D-brane configurations on an orbifold $X / G$ are classified by the $G$-equivariant $K$-theory $K_{G}(X)$ in the type IIB theory, and $K_{G}^{-1}(X)$ in the type IIA theory. Therefore, to classify stable D-brane configurations in the type II theories on the orbifold limit of $K 3, \mathbb{T}^{4} / \mathbb{Z}_{2}$, we must compute $K_{\mathbb{Z}_{2}}^{*}\left(\mathbb{T}^{4}\right)$.

For the remainder of this paper let $G=\mathbb{Z}_{2}$ and $R=R(G)=\mathbb{Z}[t] /\left(t^{2}-1\right)$ be the representation ring of $G$, where $t$ is the non-trivial character of $G$. Let $I=(t-1)$ and $J=(t+1)$. These are prime ideals with $R / I \cong R / J \cong \mathbb{Z}$, and $R_{(I)} \cong R_{(J)} \cong \mathbb{Q}$ (see Appendix).

$K_{G}(X)$ is an $R$-module, and the $R$-module structure carries more information than just the abelian group structure. For the sake of generality we compute $K_{G}^{*}\left(\mathbb{T}^{n}\right)$ as an $R$-module when $G$ acts on $\mathbb{T}^{n}=\mathbb{R}^{n} / \mathbb{Z}^{n}$ by -1 and we place no restriction on $n$.

Theorem 1. Let $G$ act on $\mathbb{T}^{n}=\mathbb{R}^{n} / \mathbb{Z}^{n}$ via multiplication by -1 on $\mathbb{R}^{n}$. Then $K_{G}^{*}\left(\mathbb{T}^{n}\right)$ is entirely concentrated in even degrees, and $K_{G}^{0}\left(\mathbb{T}^{n}\right) \cong 2^{n-1}$. $R \oplus 2^{n-1} \cdot(R / J)$. 
Proof. Throughout this proof we will be using the result from [27]: If $C$ is a closed $G$-invariant subspace of a locally compact $G$-space $X$ then the sequence

$$
\begin{aligned}
K_{G}^{0}(X-C) & \rightarrow K_{G}^{0}(X) \rightarrow K_{G}^{0}(C) \rightarrow K_{G}^{1}(X-C) \rightarrow K_{G}^{1}(X) \\
& \rightarrow K_{G}^{1}(C) \rightarrow K_{G}^{0}(X-C) \rightarrow \cdots
\end{aligned}
$$

is exact. Here the last part was gotten using Bott periodicity, $K_{G}^{2}(X-C) \cong$ $K_{G}^{0}(X-C)$. We will also use the result from [10]:

$$
\begin{aligned}
K_{G}^{0}(\mathrm{pt})=K_{G}^{0}\left(\mathbb{R}^{k}\right) & =R, \text { if } k \text { is even, } \\
K_{G}^{0}\left(\mathbb{R}^{k}\right) & =R / J, \text { if } k \text { is odd, } \\
K_{G}^{1}(\mathrm{pt})=K_{G}^{1}\left(\mathbb{R}^{k}\right) & =0 .
\end{aligned}
$$

A fundamental domain for $\mathbb{T}^{n}$ is $F=\left\{\left(x_{1}, \ldots, x_{n}\right):\left|x_{j}\right| \leq \frac{1}{2}\right\} / \sim$, where $-\frac{1}{2} \sim \frac{1}{2}$. Define:

$$
\begin{aligned}
& Y_{k}=\bigcup_{i_{n-k}=i_{n-k-1}+1}^{n} \cdots \bigcup_{i_{2}=i_{1}+1}^{n-k+2} \bigcup_{i_{1}=1}^{n-k+1}\left\{\left(x_{1}, \ldots, x_{n}\right):\right. \\
& \left.x_{i_{l}}= \pm \frac{1}{2} \text { for } 1 \leq l \leq(n-k) \text { and }\left|x_{j}\right| \leq \frac{1}{2} \text { if } j \neq i_{l}\right\} / \sim .
\end{aligned}
$$

So $Y_{k}$ is the set of all $n$-tuples where at least $n-k$ coordinates are exactly $\pm \frac{1}{2}$. Note that $Y_{k}$ is the union of $\left(\begin{array}{l}n \\ k\end{array}\right)$ copies of $\mathbb{T}^{k}$, whose pairwise intersections are all $\mathbb{T}^{k-1}$. The union of all the pairwise intersections is $Y_{k-1}$. Now by induction on $k$ we will show that $K_{G}^{0}\left(Y_{k}\right)$ is given by

$$
\left(\begin{array}{l}
n \\
k
\end{array}\right) R \oplus\left(\begin{array}{c}
n \\
k-1
\end{array}\right) R / J \oplus\left(\begin{array}{c}
n \\
k-2
\end{array}\right) R \oplus \cdots \oplus\left(\begin{array}{c}
n \\
1
\end{array}\right) R / J \oplus R
$$

if $k$ is even, and is given by

$$
\left(\begin{array}{l}
n \\
k
\end{array}\right) R / J \oplus\left(\begin{array}{c}
n \\
k-1
\end{array}\right) R \oplus\left(\begin{array}{c}
n \\
k-2
\end{array}\right) R / J \oplus \cdots \oplus\left(\begin{array}{c}
n \\
1
\end{array}\right) R / J \oplus R
$$

if $k$ is odd. We will also show that $K_{G}^{1}\left(Y_{k}\right)=0$ in both cases.

Note that $Y_{0}=\mathrm{pt}$, so $K_{G}^{*}\left(Y_{0}\right)=R$, all in degree 0 . Let us now look at the case of $k=1$. $Y_{1}$ is the one-point union of $\left(\begin{array}{l}n \\ 1\end{array}\right)=n 1$-tori. The point of 
intersection, $y=\left( \pm \frac{1}{2}, \pm \frac{1}{2}, \ldots, \pm \frac{1}{2}\right)$, is a closed $G$-invariant subset of $Y_{1}$, so by (3) we get an exact sequence

$$
K_{G}^{0}\left(Y_{1} \backslash\{y\}\right) \rightarrow K_{G}^{0}\left(Y_{1}\right) \rightarrow K_{G}^{0}(\mathrm{pt}) \rightarrow K_{G}^{1}\left(Y_{1} \backslash\{y\}\right) \rightarrow K_{G}^{1}\left(Y_{1}\right) \rightarrow K_{G}^{1}(\mathrm{pt}) .
$$

$Y_{1} \backslash\{y\}$ is the disjoint union of $n$ copies of $\mathbb{R}$. Using this and the above exact sequence we can see immediately that $K_{G}^{1}\left(Y_{1}\right)=0$ since both $K_{G}^{1}(\mathbb{R})$ and $K_{G}^{1}(\mathrm{pt})$ are 0 by (4). Moreover, we get a short exact sequence

$$
0 \longrightarrow n(R / J) \longrightarrow K_{G}^{0}\left(Y_{1}\right) \longrightarrow R \longrightarrow 0
$$

which splits since $R$ is free. So $K_{G}^{0}\left(Y_{1}\right)=n(R / J) \oplus R$.

Now let us look at the inductive step. Assume the above formula for $K_{G}^{*}\left(Y_{k}\right)$ holds for all $k<m$. Let us also assume $m$ is even. $Y_{m-1}$ is a closed $G$-invariant subset of $Y_{m} . Y_{m} \backslash Y_{m-1} \cong\left(\begin{array}{c}n \\ m\end{array}\right) \mathbb{R}^{m}$, since it is the set of all $n$-tuples with $n-m$ components exactly $\pm \frac{1}{2}$ and $m$ components with absolute value strictly less than $\frac{1}{2}$. Therefore $K_{G}^{*}\left(Y_{m} \backslash Y_{m-1}\right) \cong\left(\begin{array}{c}n \\ m\end{array}\right) R$ all in degree 0 , by (4) since $m$ is even. Also, by the inductive assumption and since $m-1$ is odd, $K_{G}^{*}\left(Y_{m-1}\right) \cong\left(\begin{array}{c}n \\ m-1\end{array}\right) R / J \oplus\left(\begin{array}{c}n \\ m-2\end{array}\right) R \oplus\left(\begin{array}{c}n \\ m-3\end{array}\right) R / J \oplus \cdots \oplus$ $\left(\begin{array}{l}n \\ 1\end{array}\right) R / J \oplus R$ all in degree zero. By (3) we see that $K_{G}^{1}\left(Y_{m}\right)=0$ and we get a short exact sequence:

$$
0 \rightarrow K_{G}^{0}\left(Y_{m} \backslash Y_{m-1}\right) \rightarrow K_{G}^{0}\left(Y_{m}\right) \rightarrow K_{G}^{0}\left(Y_{m-1}\right) \rightarrow 0 .
$$

By Proposition A.5 of the Appendix, the exact sequence splits and we see that

$$
\begin{aligned}
K_{G}^{*}\left(Y_{m}\right) & \cong K_{G}^{*}\left(Y_{m} \backslash Y_{m-1}\right) \oplus K_{G}^{*}\left(Y_{m-1}\right) \\
& \cong\left(\begin{array}{c}
n \\
m
\end{array}\right) R \oplus\left(\begin{array}{c}
n \\
m-1
\end{array}\right) R / J \oplus\left(\begin{array}{c}
n \\
m-2
\end{array}\right) R \oplus \cdots \oplus\left(\begin{array}{c}
n \\
1
\end{array}\right) R / J \oplus R,
\end{aligned}
$$

all in degree 0 . The inductive step for $m$ odd follows the same form. Note that $Y_{n}$ is the entire space $\mathbb{T}^{n}$, so by the above inductive proof we have shown:

$$
\begin{aligned}
K O_{G}^{0}\left(\mathbb{T}^{n}\right) & \cong\left\{\begin{array}{l}
R \oplus\left(\begin{array}{c}
n \\
n-1
\end{array}\right) R / J \oplus\left(\begin{array}{c}
n \\
n-2
\end{array}\right) R \oplus \cdots \oplus\left(\begin{array}{l}
n \\
1
\end{array}\right) R / J \oplus R, \text { if } n \text { is even } \\
R / J \oplus\left(\begin{array}{c}
n \\
n-1
\end{array}\right) R \oplus\left(\begin{array}{c}
n \\
n-2
\end{array}\right) R / J \oplus \cdots \oplus\left(\begin{array}{c}
n \\
1
\end{array}\right) R / J \oplus R, \text { if } n \text { is odd }
\end{array}\right. \\
& \cong \sum_{j \leq n \text { even }}\left(\begin{array}{c}
n \\
j
\end{array}\right) R \oplus \sum_{j \leq n, \text { odd }}^{n}\left(\begin{array}{c}
n \\
j
\end{array}\right) R / J
\end{aligned}
$$


Note that $0=(1-1)^{n}=\sum_{j \leq n \text { even }}\left(\begin{array}{c}n \\ j\end{array}\right)-\sum_{j \leq n \text { odd }}\left(\begin{array}{l}n \\ j\end{array}\right)$, which implies

$$
\begin{aligned}
2^{n} & =(1+1)^{n}=\sum_{j=0}^{n}\left(\begin{array}{l}
n \\
j
\end{array}\right) \\
& =\sum_{j \leq n \text { even }}\left(\begin{array}{l}
n \\
j
\end{array}\right)+\sum_{j \leq n \text { odd }}\left(\begin{array}{l}
n \\
j
\end{array}\right) \\
& =2 \sum_{j \leq n \text { even }}\left(\begin{array}{l}
n \\
j
\end{array}\right) .
\end{aligned}
$$

Therefore, we see that $\sum_{j \leq n \text { even }}\left(\begin{array}{l}n \\ j\end{array}\right)=\sum_{j \leq n \text { odd }}\left(\begin{array}{l}n \\ j\end{array}\right)=2^{n-1}$. Putting this into (6) gives us our final result:

$$
K_{G}^{*}\left(\mathbb{T}^{n}\right) \cong 2^{n-1} \cdot R \oplus 2^{n-1} \cdot(R / J)
$$

all in degree zero.

When $n$ is even, Theorem 1 classifies stable $D$-branes on the orbifold $\mathbb{T}^{n} / \mathbb{Z}_{2}$ in the two type II theories. When $n$ is odd, the action of $\mathbb{Z}_{2}$ on $\mathbb{T}^{n}$ reverses orientation, so we cannot define an oriented string theory on $\mathbb{T}^{n} / \mathbb{Z}_{2}$. In order to get a consistent string theory we would also have to mod out by the action of the worldsheet parity operator to obtain unoriented strings. Stable $D$-brane configurations would then be classified by $K R$-theory [37].

Returning to the case of $n=4$, the orbifold limit of $K 3$, we find

$$
K_{G}^{0}\left(\mathbb{T}^{4}\right)=R^{8} \oplus(R / J)^{8}
$$

which as an abelian group is $\mathbb{Z}^{24}$. This result is consistent with the Localization Theorem (Theorem A.1), which says that $K_{G}^{0}\left(\mathbb{T}^{4}\right)_{(J)} \cong K_{G}^{0}\left(\left(\mathbb{T}^{4}\right)^{G}\right)_{(J)} \cong$ $\left(K_{G}^{0}(\mathrm{pt})^{16}\right)_{(J)}$, since $R_{(J)} \cong(R / J)_{(J)} \cong K_{G}(\mathrm{pt})_{(J)}$. Note that the equivariant $K$-theory of $\mathbb{T}^{4}$ is isomorphic to the $K$-theory of $K 3$ as an abelian group, but has the added benefit of an $R$-module structure on it. Using the $R$-module structure of $K_{G}^{*}\left(\mathbb{T}^{4}\right)$ as well as some facts about $G$-equivariant $K$-theory and the homological algebra of $R(G)$ given in the Appendix, we can now determine the $R$-module structure on $K_{G}^{*}(M, \partial M) \cong K^{*}(N, \partial N)$. The following is a refinement of the results of Section 3, and can be viewed as the main mathematical result of this paper. 
Theorem 2. As before, let $G=\mathbb{Z}_{2}$ act on $\mathbb{T}^{4}=\mathbb{R}^{4} / \mathbb{Z}^{4}$ by multiplication by -1 on $\mathbb{R}^{4}$, and let $M$ be the result of removing 16 open balls from $\mathbb{T}^{4}$, one ball around each fixed point of the G-action. Then

$$
K_{G}^{0}(M, \partial M) \cong(R / I)^{7}, \quad K_{G}^{1}(M, \partial M) \cong(R / I)^{10} \oplus(R / 2 I)^{5}
$$

as $R$-modules.

Proof. With notation as before, $(N, \partial N)$ is obtained from $(M, \partial M)$ by dividing out by a free $\mathbb{Z}_{2}$-action, so by Theorem A.1

$$
K_{G}^{*}(M, \partial M) \cong K^{*}(N, \partial N)
$$

as $\mathbb{Z}$-modules. Furthermore, Theorem A.1 tells us that $K_{G}^{*}(M, \partial M)$ localized at $J$ must be zero. This along with the result $K^{0}(N, \partial N) \cong \mathbb{Z}^{7}$ as a $\mathbb{Z}$-module, found in the previous section, shows that as an $R$-module,

$$
K_{G}^{0}(M, \partial M) \cong(R / I)^{7}
$$

by using Propositions A.2 and A.4 from the Appendix. Alternatively, the $R$-module structure on $K_{G}^{0}(M, \partial M)$ is determined by the action of $t$, which by Proposition A.1 from the Appendix is given by cup-product with the class of a line bundle in $K^{0}(N)$, whose first Chern class is torsion. Since $K^{0}(N, \partial N)$ is torsion-free, this action has to be trivial.

Since $K_{G}^{1}(M, \partial M) \cong K^{1}(N, \partial N) \cong \mathbb{Z}^{15} \oplus \mathbb{Z}_{2}^{5}$ as a $\mathbb{Z}$-module, and must be zero when localized at $J$, it is an extension of $(R / I)^{15}$ by $(R /(2, I))^{5}$, and the possible $R$-module structures are:

$$
(R / I)^{15-k} \oplus(R /(2, I))^{5-k} \oplus(R / 2 I)^{k},
$$

where $0 \leq k \leq 5$. There are a few ways to determine the value of $k$. One method is to use the long exact sequence induced on equivariant $K$-theory for the pair $\left(\mathbb{T}^{4}, \partial M\right)$ :

$$
\begin{aligned}
& \cdots \underset{\rightarrow}{\rightarrow} K_{G}^{0}\left(\mathbb{T}^{4}, \partial M\right) \stackrel{\alpha}{\rightarrow} K_{G}^{0}\left(\mathbb{T}^{4}\right) \stackrel{\beta}{\rightarrow} K_{G}^{0}(\partial M) \\
& \stackrel{\gamma}{\rightarrow} K_{G}^{1}\left(\mathbb{T}^{4}, \partial M\right) \rightarrow K_{G}^{1}\left(\mathbb{T}^{4}\right) \rightarrow \cdots .
\end{aligned}
$$

By excision,

$$
\begin{aligned}
K_{G}^{*}\left(\mathbb{T}^{4}, \partial M\right) & \cong K_{G}^{*}\left(\mathbb{T}^{4}-\partial M\right) \\
& \cong K_{G}^{*}(\operatorname{int} M) \oplus K_{G}^{*}\left(16 \mathbb{R}^{4}\right) \\
& \cong K_{G}^{*}(M, \partial M) \oplus\left(K_{G}^{*}\left(\mathbb{R}^{4}\right)\right)^{16}
\end{aligned}
$$


$K_{G}^{*}\left(\mathbb{R}^{4}\right)$ is given by equation (4) and is all in degree zero, so

$$
K_{G}^{1}(M, \partial M) \cong K_{G}^{1}\left(\mathbb{T}^{4}, \partial M\right) .
$$

The $R$-module structure of $K_{G}^{*}(\partial M) \cong K_{G}^{*}\left(16 S^{3}\right)$ is given in Example A.1. Plugging this, as well as $K_{G}^{*}\left(\mathbb{T}^{4}\right)$ from Theorem 1, into the long exact sequence (11), we get the exact sequence

$$
\begin{aligned}
0 \rightarrow & (R / I)^{16} \rightarrow R^{16} \oplus(R / I)^{7} \stackrel{\alpha}{\rightarrow} R^{8} \oplus(R / J)^{8} \\
& \stackrel{\beta}{\rightarrow}(R / 2 I)^{16} \stackrel{\gamma}{\rightarrow} K_{G}^{1}(M, \partial M) \rightarrow 0
\end{aligned}
$$

or

$$
0 \rightarrow(\operatorname{coker} \alpha \cong \operatorname{im} \beta) \rightarrow(R / 2 I)^{16} \stackrel{\gamma}{\rightarrow} K_{G}^{1}(M, \partial M) \rightarrow 0 .
$$

Now $\operatorname{im} \beta$ contains the diagonal copy $\Delta(R / 2 I)$ of $R / 2 I$ inside $(R / 2 I)^{16}$. One can see this as follows: the trivial line bundle $1_{\mathbb{T}^{4}}$ on $\mathbb{T}^{4}$ restricts to the trivial line bundle on each component of $\partial M$, or in other words to $\Delta(\mathrm{i})$, where $\mathrm{i}$ is the image of 1 in $R / 2 I$. Since $R / 2 I$ is generated as an $R$-module by $\dot{1}$ and $r \cdot 1_{\mathbb{T}^{4}}$ restricts to $\Delta(r \cdot \dot{1})$ for $r \in R, \Delta(R / 2 I) \subseteq \operatorname{im} \beta$. Since $(R / 2 I)^{16} / \Delta(R / 2 I) \cong(R / 2 I)^{15}, K_{G}^{1}(M, \partial M)$ is a quotient of $(R / 2 I)^{15}$. Therefore, it has at most 15 cyclic summands as an $R$-module, so $(15-k)+$ $(5-k)+k=20-k \leq 15$ and $k=5$, which completes the proof.

Since $K^{*}(N)$ is used to classify $D$-brane charges in the type IIB theory, we can also compute the absolute (i.e., not rel boundary) equivariant $K$-theory of $M$.

Theorem 3. Using the same notation as above

$$
K_{G}^{0}(M) \cong(R / 2 I) \oplus(R /(2, I))^{4} \oplus(R / I)^{6}, \quad K_{G}^{-1}(M) \cong(R / I)^{15}
$$

as $R$-modules.

Proof. We start with equation (1), which determines $K_{G}^{*}(M)$ as a $\mathbb{Z}$-module. Just as in the proof above, we immediately conclude that $K_{G}^{-1}(M) \cong$ $(R / I)^{15}$ and that

$$
K_{G}^{0}(M) \cong(R / I)^{7-k} \oplus(R /(2, I))^{5-k} \oplus(R / 2 I)^{k},
$$

where $0 \leq k \leq 5$. To finish the proof, we can use Proposition A.1 of the Appendix. Since $N=M / G$ has all its even-dimensional cohomology in degrees 0 and 2, the Chern character is a ring isomorphism 
$K^{0}(N) \rightarrow \mathbb{Z} \oplus H^{2}(N, \mathbb{Z})$, with $H^{2}(N, \mathbb{Z})$ an ideal whose square is zero, and the action of $t$ corresponds to multiplication by $1+c$, where $c \in$ $H^{2}(N, \mathbb{Z})$ corresponds to the two-fold covering map $M \rightarrow M / G$. Note that $\mathbb{Z} \cdot 1+\mathbb{Z}_{2} \cdot c$ is a subring of $K^{0}(N)$ isomorphic to $R / 2 I$, and Proposition A.1 says that the $R$-module structure on $K_{G}^{0}(M)$ comes from the action of this subring. However, one can also see that it is impossible to get two elements of $K_{G}^{0}(M)$, each with annihilator $2 I$, which are linearly independent over $R / 2 I$. For suppose such elements had the form $n_{j}+x_{j}$, with $n_{j} \in \mathbb{Z}$ and $x_{j} \in H^{2}(N, \mathbb{Z})$. The condition that the annihilator of $n_{j}+x_{j}$ is $2 I$ means $n_{j}$ is odd. However, then $n_{1}+n_{2}$ is even, so the sum of the two elements is annihilated by $I$, and so they are not linearly independent over $R / 2 I$. So $K_{G}^{0}(M)$ cannot have more than one summand isomorphic to $R / 2 I$.

\section{Conclusion}

We have seen that the group that classifies the BPS D-branes in type-I superstring theory compactified on $\mathbb{T}^{4}, K O\left(\mathbb{T}^{4} \times \mathbb{R}^{6}\right)$, is isomorphic to $\mathbb{Z}^{6} \oplus$ $\mathbb{Z}_{2}{ }^{5}$, which injects into both $K^{-1}\left((N, \partial N) \times \mathbb{R}^{6}\right) \cong \mathbb{Z}^{15} \oplus \mathbb{Z}_{2}{ }^{5}$ and $K^{0}(N \times$ $\left.\mathbb{R}^{6}\right)$ with an isomorphism on the torsion. The isomorphism on torsion is significant because the torsion brane charges occurring in the type I theory can only be explained by $D$-branes carrying torsion charge in the type II theories and could not be explained by the $K$-theory of $K 3$. There would also be no way to obtain torsion charges from charge groups for RR or NS-NS fields living in cohomology of $K 3$, since once again this is torsion-free. To understand the difference in the free ranks, we must first understand which integral brane charges we expect to correspond to stable $D$-branes in the different theories. We present two possible avenues of future research to explain the differences in the free rank.

One possibility is that we could need to include extra $\mathbb{Z}$ summands that do not appear in $K O^{-6}\left(\mathbb{T}^{4}\right)$ corresponding to other non-supersymmetric branes in type I on $\mathbb{T}^{4}$ (such as those discussed in [11]), since these would have $K$-theoretic charges living in the other groups listed in Table 1. For example, $D 6$-branes with real Chan-Paton bundles should have charges living in $K O^{-3}\left(\mathbb{T}^{4}\right) \cong \mathbb{Z}^{4}$. It is unknown which non-BPS branes in the type I theory transform to BPS branes in the type IIA theory. Further research into this phenomenon needs to be done before completing the classification. A correct classification of the BPS brane charges on the type IIA side is a powerful tool when studying non-BPS branes that map to BPS branes. If we knew all of the stable brane charges in the type IIA theory, it would give an upper bound on the additional stable non-BPS branes we must include in our classification of type I $D$-branes by the duality. It is important to note 
"ATMP-16-6-A1-MEN" — 2013/5/22 — 21:05 — page 1608 — \#18

that it would not be enough to just know all of the stable brane charges in the type IIA theory, but we would also need to know which branes carry RR charge and which carry NS-NS charge. However, if we knew all of the stable brane charges and had a correct classification of the $D$-brane charges via $K$-theory, by elimination we would know the stable NS-NS charges as well.

To understand which $D$-branes should be included in the classification, we believe the next step is to determine exactly what cycles a $D$-brane can wrap. In the literature, when analyzing different aspects of this duality, different authors have used both a smooth $K 3$ and the orbifold limit, $\mathbb{T}^{4} / \mathbb{Z}_{2}$. In our calculation, we combined different features from a smooth $K 3$ and $\mathbb{T}^{4} / \mathbb{Z}_{2}$. By looking at features from both points in the moduli space we were able to describe the torsion brane charges successfully. To fully understand the discrepancy in the free rank, one needs to determine which cycles come from which description and which ones should be included in the classification.

The type IIA theory on $K 3$ has 24 gauge bosons, all coming from the RR sector. 22 of them correspond to reductions of the type IIA RR 3-form potential on 2-cycles of $K 3$. Of these, 19 correspond to anti-self-dual forms, and 16 of those map to the 16 gauge bosons in the heterotic theory that come from the Cartan subalgebra of the rank-16 gauge group [1, p. 11]. In the type IIA theory at the orbifold limit, these 16 gauge bosons correspond to $D 2$-branes wrapping 2-cycles at the singularities. The 2-cycles correspond to the blow-up of the orientifold fixed points and go to zero size at the orbifold point. While the sizes of the 2 -cycles go to zero, the $D$-brane tensions remain finite [22]. These $16 \mathrm{D} 2$-branes are supersymmetric since the cycles they wrap are. When the radius of the circle of the original torus that passes through two fixed points is smaller than a certain critical radius, the two BPS D2-branes associated to the fixed points will decay into a single stable non-BPS $D 2$-brane. The single non-BPS $D 2$-brane can be viewed as wrapped around a single 2-cycle of $K 3$ that is homologically equivalent to the sum of the supersymmetric 2-cycles corresponding to the two fixed points [22]. In this region, the non-BPS brane has a lower mass than that of the combined system of two BPS branes, so the non-BPS brane configuration is the stable one. Performing a $T$-duality transformation sends these $D 2$ branes to $D$-strings between the different $N S 5$-branes in the type IIB theory, and sends the blow-up modes that determine the 2-cycles in the type IIA case to moduli determining the location of the $N S 5$-branes $[1,22]$. The locations of the NS5 branes affect the mass of the $D$-string configurations (and hence the $D 2$-branes in the IIA theory, by $T$-duality), since changing the locations of the NS5-branes changes the lengths of the strings [22]. In our computation of stable $D$-brane charges in the type IIB theory, we moved the NS5-branes away from the orbifold fixed points, changing the mass of the BPS brane system. However, in the type IIA theory we looked at the 
interior of $N$. When we excise the fixed points, the 2-cycles that shrink to size zero at the orbifold points can no longer shrink to zero size in $\stackrel{\circ}{N}$. In

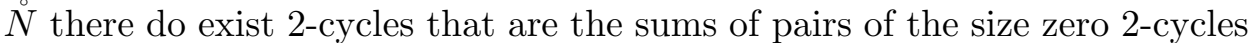
with a factor of a half as can be seen by looking at the Kummer lattice. More research needs to be performed to determine if we want to include the charges from the BPS branes or the non-BPS branes. Note that since a single non-BPS brane corresponds to 2 BPS branes, this could possibly reduce the free rank of the $K$-theoretic classification of $D$-brane charges in the two type II theories. The $R(G)$-module structure of $K_{\mathbb{Z}_{2}}^{*}(M, \partial M)$ and $K_{\mathbb{Z}_{2}}^{*}(M)$ provides added constraints (beyond those coming from the abelian group structure) that can be used to complete this determination, since it provides added information about where different charges in $N$ come from in relation to the orbifold $\mathbb{T}^{4} / \mathbb{Z}_{2}$.

Another benefit of Theorems 1 and 2 becomes apparent when trying to include a twisting due to an $H$-flux. On an orbifold $X / G$, it is unclear what is meant by the $H$-flux. It does not make sense for $H$ to live in $H^{3}(X ; \mathbb{Z})$ since to make any sense on the orbifold, $H$ would have to be $G$ invariant. Recent work by Distler et al. in [9] proposes using more exotic twistings involving equivariant cohomology, but the precise definition of the $H$-flux on an orbifold remains an open problem. Knowing the equivariant structures of $K_{\mathbb{Z}_{2}}^{*}(M, \partial M)$ and $K_{\mathbb{Z}_{2}}^{*}\left(\mathbb{T}^{n}\right)$ allows us to apply twistings that take advantage of the equivariant structure. Furthermore, when $n=4$, Theorem 1 shows that considering $\mathbb{Z}_{2}$-equivariant states on the orbifold limit of $K 3$ gives a $D$-brane spectrum equivalent to the one arising from considering states on $K 3$ itself. Note that there can be no non-trivial $H$-flux on $K 3$ (since $H^{3}(K 3)=0$ ), but it may be possible to apply a twisting such as one of the ones proposed in [9] or [7] to $K_{\mathbb{Z}_{2}}^{*}\left(\mathbb{T}^{4}\right)$.

While showing that the stable $D$-brane configurations in the two theories match does not prove the two theories are dual, this particular example illustrates how ensuring that stable $D$-brane configurations match is a useful first step in checking a possible duality. By looking at the stable $D$-branes in this case we saw immediately that we did not want to use $K 3$, but rather a desingularized version of the orbifold blow-down, as matched with our physical intuition. Furthermore, the $K$-theoretic classification of $D$-branes provides useful tools for studying other phenomena that arise in string theory dualities, such as non-BPS branes that map to BPS branes.

This example also illustrates the benefit of composing known dualities to gain information about less understood dualities. By composing a known duality between the type I and $\mathrm{SO}(32)$ heterotic theories with one between the $\mathrm{SO}(32)$ heterotic and type IIA theories to obtain a duality between the type I and type IIA theories, and then composing that with $T$-duality 
"ATMP-16-6-A1-MEN" — 2013/5/22 — 21:05 — page 1610 — \#20

between type IIA and IIB, we were able to come up with examples of dualities between all 5 superstring theories. Knowing what the theory looks like in all of the superstring theories provides more information to use when testing possible dualities.

\section{Acknowledgments}

The Research of the first author was partially supported by NSF grants DMS-0504212 and DMS-0805003. Some of this work constituted part of the author's PhD dissertation, submitted to the University of Maryland in May, 2010. The research of the second author was partially supported by NSF grants DMS-0504212 and DMS-0805003.

\section{Appendix A Some homological algebra over $R\left(\mathbb{Z}_{2}\right)$}

This appendix collects together some facts about the homological algebra of the representation $\operatorname{ring} R=R(G)$ for $G=\mathbb{Z}_{2}$, which are useful for studying $G$-equivariant $K$-theory. The connection is that $G$-equivariant $K$-groups are always modules over $R=R(G)$, and the $R$-module structure carries more information than just the abelian group structure of the $K_{G \text {-groups. The }}$ basic reference for representation rings is [28] and the basic reference for equivariant $K$-theory is [27].

Recall that for a compact group $G, R(G)$ is the free abelian group on the equivalence classes of irreducible (finite-dimensional complex) representations of $G$, with multiplication coming from the tensor product of representations. When $G$ is also abelian, $R(G)$ is just the group ring of the Pontrjagin dual group $\widehat{G}$. In what follows, we always take $G=\mathbb{Z}_{2}$, $R=R(G)=\mathbb{Z}[t] /\left(t^{2}-1\right)$. The generator $t$ corresponds to the non-trivial character of $G$. The ring $R$ has two important prime ideals, $I=(t-1)$ (the augmentation ideal) and $J=(t+1)$. These play symmetrical roles since there is an automorphism of $R$ (not coming from an automorphism of $G$ ) which interchanges them. In the sense of [28], $I$ has support $\{0\}$ (i.e., just the identity element) and $J$ has support $G$. If we localize $R$ at $I$, we get a local ring $R_{(I)}$ in which everything not in $I$ is invertible. In particular, every prime $p \in \mathbb{N}$ is inverted, so $R_{(I)}$ is a $\mathbb{Q}$-vector space. However, $R \otimes \mathbb{Q}$ splits as a direct sum $\mathbb{Q} \oplus \mathbb{Q}$, with $t$ acting by 1 on one factor and by -1 on the other factor. Since $t+1$ must be invertible on $R_{(I)}$, the summand where $t$ acts by -1 must die and so $R_{(I)} \cong \mathbb{Q}$ with $t$ acting by +1 . Similarly, $R_{(J)} \cong \mathbb{Q}$ with $t$ acting by -1 . Furthermore, we have $I \cdot J=0, I=\operatorname{Ann}_{R}(J), J=\operatorname{Ann}_{R}(I)$, 
$R / I \cong J$ (as $R$-modules), and $R / J \cong I$ (as $R$-modules). Also, $(R / J)_{(I)}=0$, $(R / I)_{(J)}=0,(R / J)_{(J)} \cong R_{(J)}$, and $(R / I)_{(I)} \cong R_{(I)}$. But $I+J$ is a proper $R$-submodule of $R$ (of index 2 ).

The Segal Localization Theorem [27, Proposition 4.1] specializes to the following:

Theorem A.1 (Segal). Let $G=\mathbb{Z}_{2}$ and let $X$ be a locally compact $G$-space. Then $K_{G}^{*}(X)_{(J)} \cong K^{*}\left(X^{G}\right) \otimes_{\mathbb{Z}} \mathbb{Q}$, with $t$ acting by -1 . In particular, if $G$ acts freely on $X$, then $K_{G}^{*}(X)_{(J)}=0$, and $K_{G}^{*}(X) \cong K^{*}(X / G)$, at least as $\mathbb{Z}$-modules.

When $G$ acts freely on $X$, one can make Theorem A.1 a bit more precise.

Proposition A.1. Let $G=\mathbb{Z}_{2}$ and let $X$ be a compact free $G$-space. Then the $R(G)$-module structure on $K_{G}^{*}(X) \cong K^{*}(X / G)$ is defined by letting $t$ act by tensoring with the line bundle $V$ with $c_{1}(V)=c$, where $c$ is the image in $H^{2}(X / G, \mathbb{Z})$ under the Bockstein homomorphism of the class in $H^{1}\left(X / G, \mathbb{Z}_{2}\right)$ classifying the 2 -to-1 covering map $X \rightarrow X / G$. (If $X$ is a connected reasonable space, such as a manifold, then $H^{1}\left(X / G, \mathbb{Z}_{2}\right) \cong$ $\operatorname{Hom}\left(\pi_{1}(X / G), \mathbb{Z}_{2}\right)$ classifies $2-$ to-1 covering spaces of $X / G$, by covering space theory. One can also realize $V$ more explicitly as the fiber product $X \times_{G} \mathbb{C}$, where $G$ acts on $\mathbb{C}$ by the non-trivial character $t$.)

If $A$ is a closed $G$-invariant subspace of $X$, then the $R(G)$-module structure on

$$
K_{G}^{*}(X, A) \cong K^{*}(Y, B), \quad Y=X / G, B=A / G,
$$

is again defined by letting $t$ act by cup-product with $[V] \in K^{0}(X / G)$. (Recall that for any pair $(Y, B)$, we have the cup-product $K^{0}(Y) \otimes K^{*}(Y, B) \rightarrow$ $K^{*}(Y, B)$.)

Proof. The definition of the $R(G)$-action on $K_{G}^{*}(X)$ or on $K_{G}^{*}(X, A)$ implies that the result of applying the module action of $t$ corresponds to tensoring with $(\mathbb{C}, t)$, which is the same after applying the isomorphisms $K_{G}^{*}(X) \cong$ $K^{*}(Y)$ or $K_{G}^{*}(X, A) \cong K^{*}(Y, B)$ as taking the vector bundle tensor product with $V$. The rest is immediate.

Corollary A.1. If $G=\mathbb{Z}_{2}$ and $X$ is a compact free $G$-space, then the $R$-module structure on $K^{*}(X / G)$ is trivial (i.e., factors through $R / I$ ) if and only if $c=0$ in $H^{2}(X / G, \mathbb{Z})$ (in the notation of the Proposition). 
Proof. If $c=0$ in $H^{2}(X / G, \mathbb{Z})$, then (in the notation of Proposition A.1) $V$ is the trivial bundle and the action of $t$ is trivial. But if $c \neq 0$, then $t \cdot 1=[V] \neq 1$, so the action is non-trivial.

Example A.1. Let $S^{3}$ be given the antipodal action of $G$. Then $K_{G}^{0}\left(S^{3}\right) \cong$ $R / 2 I$ and $K_{G}^{1}\left(S^{3}\right) \cong R / I$. Indeed, we know that $K_{G}^{0}\left(S^{3}\right) \cong K^{0}\left(S^{3} / G\right)=$ $K^{0}\left(\mathbb{R P}^{3}\right) \cong \mathbb{Z} \oplus \mathbb{Z} / 2$ as an abelian group, but by Corollary A.1, the $R$ module structure must be non-trivial, so this follows from Proposition A.8 below. The $R$-module structure on $K_{G}^{1}\left(S^{3}\right) \cong K^{1}\left(S^{3} / G\right)=K^{1}\left(\mathbb{R P}^{3}\right) \cong \mathbb{Z}$ must be trivial by Proposition A.2 below. Alternatively, one can compute directly from the exact sequence

$$
\begin{aligned}
0= & K_{G}^{1}\left(D^{4}\right) \rightarrow K_{G}^{1}\left(S^{3}\right) \rightarrow K_{G}^{0}\left(\operatorname{int} D^{4}\right) \\
& \stackrel{\beta}{\rightarrow} K_{G}^{0}\left(D^{4}\right) \rightarrow K_{G}^{0}\left(S^{3}\right) \rightarrow K_{G}^{1}\left(\operatorname{int} D^{4}\right)=0 .
\end{aligned}
$$

We have $K_{G}^{0}\left(D^{4}\right) \cong K_{G}^{0}(\mathrm{pt})=R$ since $D^{4}$ is equivariantly contractible, and $K_{G}^{0}\left(\right.$ int $\left.D^{4}\right) \cong R$ by equivariant Bott periodicity. So one only needs to compute the map $\beta$, which is multiplication by the Bott element. This is the exterior algebra complex of $(\mathbb{C}, t)^{2}$, which is $2(1-t)$. So the image of $\beta$ is exactly $2 I$, and the kernel of $\beta$ is $\operatorname{Ann}_{R}(2 I)=J \cong R / I$.

We now need some facts about certain special $R$-modules.

Proposition A.2. Let $M$ be an $R$-module which as a $\mathbb{Z}$-module is isomorphic to $\mathbb{Z}$. Then either $M \cong R / I$ or $M \cong R / J$.

Proof. The $R$-module structure is determined by the action of $t$, which must be an automorphism of $M$ as a $\mathbb{Z}$-module. Since $\operatorname{Aut}_{\mathbb{Z}}(\mathbb{Z})=G L(1, \mathbb{Z})=$ $\{1,-1\}$, there are exactly two possibilities: $R / I$ if $t$ acts by +1 , and $R / J$ if $t$ acts by -1 .

Proposition A.3. Let $M$ be an $R$-module which as a $\mathbb{Z}$-module is isomorphic to $\mathbb{Z}_{2}$. Then $M \cong R /(2, I) \cong I / 2 I$.

Proof. The $R$-module structure is determined by the action of $t$, which must be an automorphism of $M$ as a $\mathbb{Z}$-module. But $\mathbb{Z}_{2}$ has no non-trivial automorphisms, so there is only the trivial possibility.

Proposition A.4. $\operatorname{Ext}_{R}^{1}(R / I, R / I)=0$, and thus every $R$-module extension of $R / I$ by $R / I$ splits. Similarly, $\operatorname{Ext}_{R}^{1}(R / J, R / J)=0$, so every $R$ module extension of $R / J$ by $R / J$ splits. 
Proof. We only do the first case, as the second is precisely analogous. Start with the extension of $R$-modules $0 \rightarrow I \rightarrow R \rightarrow R / I \rightarrow 0$, and apply $\operatorname{Hom}_{R}(\ldots, R / I)$. We get

$$
\begin{aligned}
0 & \rightarrow \operatorname{Hom}_{R}(R / I, R / I) \stackrel{\alpha}{\rightarrow} \operatorname{Hom}_{R}(R, R / I) \\
& \rightarrow \operatorname{Hom}_{R}(I, R / I) \rightarrow \operatorname{Ext}_{R}^{1}(R / I, R / I) \rightarrow \operatorname{Ext}_{R}^{1}(R, R / I)=0 .
\end{aligned}
$$

Now $\operatorname{Hom}_{R}(R, R / I) \cong R / I$, and since any $R$-module map $R \rightarrow R / I$ is determined by the image of 1 , which is annihilated by $I$, it comes from something in $\operatorname{Hom}_{R}(R / I, R / I)$. So the map $\alpha$ is an isomorphism, and $\operatorname{Ext}_{R}^{1}(R / I, R / I) \cong \operatorname{Hom}_{R}(I, R / I)$. Since $R / I \cong J$ (this follows from Proposition A.2), a homomorphism $I \rightarrow R / I$ is the same thing as a homomorphism $\varphi: I \rightarrow J$, which is determined by the image $\varphi(t-1)$ of $t-1$. But $t-1$ is annihilated by $t+1$, so $(t+1) \varphi(t-1)=0$. However, the only element of $J$ annihilated by $t+1$ is 0 , so $\varphi=0$.

Proposition A.5. $\operatorname{Ext}_{R}^{1}(R / J, R)=0$, and thus every $R$-module of extension of $R / J$ by $R$ splits. In addition, $\operatorname{Hom}_{R}(R / J, R) \cong R / J$. Similarly, $\operatorname{Ext}_{R}^{1}(R / I, R)=0$, so every $R$-module of extension of $R / I$ by $R$ splits, and $\operatorname{Hom}_{R}(R / I, R) \cong R / I$.

Proof. We only do the first case of $R / J$, as the case of $R / I$ is precisely analogous. First observe (as in the proof of Proposition A.4) that $\operatorname{Hom}_{R}(R / J, R) \cong I \cong R / J$, since a homomorphism $R / J \rightarrow R$ is completely determined by the image of the coset of 1 , which can be anything in $\operatorname{Ann}_{R}(J)=I$. Consider the short exact sequence

$$
0 \rightarrow J \rightarrow R \rightarrow R / J \rightarrow 0
$$

and apply the functor $\operatorname{Hom}_{R}(\ldots, R)$ to it. We get

$$
\begin{aligned}
0 & \rightarrow \operatorname{Hom}_{R}(R / J, R) \rightarrow \operatorname{Hom}_{R}(R, R) \rightarrow \operatorname{Hom}_{R}(J, R) \\
& \rightarrow \operatorname{Ext}_{R}^{1}(R / J, R) \rightarrow \operatorname{Ext}_{R}^{1}(R, R)=0 .
\end{aligned}
$$

We have $\operatorname{Hom}_{R}(R, R)=R$, and by the above, $\operatorname{Hom}_{R}(J, R)=J \cong R / I$. Similarly, $\operatorname{Hom}_{R}(R / J, R)=\operatorname{Hom}_{R}(I, R)=I$. So (15) becomes the sequence

$$
0 \rightarrow I \rightarrow R \rightarrow R / I \rightarrow \operatorname{Ext}_{R}^{1}(R / J, R) \rightarrow 0,
$$

and $\operatorname{Ext}_{R}^{1}(R / J, R)=0$. 
Proposition A.6. If $M$ is an $R$-module that sits in a short exact sequence

$$
0 \rightarrow R / I \rightarrow M \rightarrow R / J \rightarrow 0 \text { or } 0 \rightarrow R / J \rightarrow M \rightarrow R / I \rightarrow 0,
$$

then either $M \cong R$ or else $M \cong R / I \oplus R / J$.

Proof. Note that $R / I$ has the periodic free resolution

$$
\cdots \stackrel{t+1}{\longrightarrow} R \stackrel{t-1}{\longrightarrow} R \stackrel{t+1}{\longrightarrow} R \stackrel{t-1}{\longrightarrow} R \rightarrow R / I \rightarrow 0 .
$$

Thus $\operatorname{Ext}_{R}^{*}(R / I, R / J)$ is the cohomology of the complex

$$
R / J \stackrel{-2}{\longrightarrow} R / J \stackrel{0}{\rightarrow} R / J \stackrel{-2}{\longrightarrow} R / J \rightarrow \cdots
$$

and $\operatorname{Ext}_{R}^{1}(R / I, R / J) \cong R /(2, J)$, which has only two elements. Thus there can be at most two isomorphism classes of extensions of $R / I$ by $R / J$. Similarly for extensions of $R / J$ by $R / I$. Since $R$ and $R / I \oplus R / J$ are clearly non-isomorphic extensions of the desired form, they are the only possibilities.

Proposition A.7. The R-module extension

$$
0 \rightarrow I / 2 I \rightarrow R / 2 I \rightarrow R / I \rightarrow 0
$$

does not split.

Proof. Suppose to the contrary that we have a splitting $s: R / I \rightarrow R / 2 I$. Then $s$ is determined by $s(1+I)$, which let us say is $a+b t+2 I$. Since $s(1+$ $I)$ projects to $1+I, a+b t \in 1+I$, and can be rewritten as $1+c(1-t)$. But $t$ acts on $R / I$ by +1 , so we must have $t(1+c(1-t))-(1+c(1-t)) \in 2 I$, i.e., $(-1-2 c)(1-t) \in 2 I$. This is impossible since $-1-2 c$ is odd, not even.

Proposition A.8. $\operatorname{Ext}_{R}^{1}(R / I, R /(2, I)) \cong R /(2, I)$. Thus any $R$-module extension of $R / I$ by $R /(2, I)$ either splits or is isomorphic to $R / 2 I$. The same holds with I replaced everywhere by $J$.

Proof. First argument. Since any $\mathbb{Z}$-module extension of $\mathbb{Z}$ by $\mathbb{Z} / 2$ splits, the issue is to compute the possible $R$-module structures on $\mathbb{Z} \oplus \mathbb{Z}_{2}$, which $\bmod \mathbb{Z}$-torsion give the $R$-module $R / I$. Such structures are determined by the action of $t$. If it is trivial, the module splits as $(R / I) \oplus(R /(2, I))$. If it is not trivial, then $t$ must map $(1, \overline{0})$ to $(1, \overline{1})$, where $\overline{0}$ and $\overline{1}$ are the two elements of $\mathbb{Z}_{2}$. This is precisely the situation in the non-split extension 
$R / 2 I$ of Proposition A.7, since $t \cdot 1=1-(1-t)$, and $1-t$ represents the non-trivial class in $I / 2 I$.

Second argument. Alternatively, we can compute Ext directly, following the method used in the proof of Proposition A.4. Consider the exact sequence

$$
\begin{aligned}
0 & \rightarrow \operatorname{Hom}_{R}(R / I, R /(2, I)) \stackrel{\alpha}{\rightarrow} \operatorname{Hom}_{R}(R, R /(2, I)) \\
& \rightarrow \operatorname{Hom}_{R}(I, R /(2, I)) \rightarrow \operatorname{Ext}_{R}^{1}(R / I, R /(2, I)) \rightarrow \operatorname{Ext}_{R}^{1}(R, R /(2, I))=0
\end{aligned}
$$

Since $R /(2, I)$ is $\mathbb{Z}_{2}$ with the trivial action of $t$, it is easy to see that the three Hom groups in this sequence are all isomorphic to $\mathbb{Z}_{2}$ as abelian groups, and thus to $R /(2, I)$ as $R$-modules (by Proposition A.3). Thus, the Ext group is also isomorphic to $\mathbb{Z}_{2}$ as an abelian group, and to $R /(2, I)$ as an $R$-module.

In dealing with compact $G$-manifolds $X^{n}$ (possibly with boundary) such as $\mathbb{T}^{4}$ and $M$, it is sometimes useful to use equivariant Poincaré duality, which applies whenever the manifold has an equivariant $\operatorname{spin}^{c}$-structure (as is the case for our examples). Thus, one has isomorphisms $K_{G}^{*}(X) \cong$ $K_{n-*}^{G}(X, \partial X)$ and $K_{G}^{*}(X, \partial X) \cong K_{n-*}^{G}(X)$. At the same time, equivariant $K$-theory and equivariant $K$-homology are related by a universal coefficient theorem.

Theorem A.2 (Universal Coefficient Theorem [21]). Let $X$ be a locally compact $G$-space. Then there is a short exact sequence of $R$-modules

$$
0 \rightarrow \operatorname{Ext}_{R}^{1}\left(K_{G}^{*+1}(X), R\right) \rightarrow K_{*}^{G}(X) \rightarrow \operatorname{Hom}_{R}\left(K_{G}^{*}(X), R\right) \rightarrow 0,
$$

which does not necessarily split.

Example A.2. Let us continue with Example A.1 about $S^{3}$ with the antipodal action. Since $\operatorname{dim} S^{3}$ is odd, Poincaré duality gives $R / 2 I \cong K_{G}^{0}\left(S^{3}\right) \cong$ $K_{1}^{G}\left(S^{3}\right)$ and $R / I \cong K_{G}^{1}\left(S^{3}\right) \cong K_{0}^{G}\left(S^{3}\right)$. Let us see that this is consistent with Theorem A.2. We have $\operatorname{Hom}_{R}(R / I, R) \cong R / I$ and $\operatorname{Ext}_{R}^{1}(R / I, R)=0$ by Proposition A.5. A homomorphism $R / 2 I \rightarrow R$ must kill the $\mathbb{Z}$-torsion $I / 2 I$ in $R / 2 I$, hence must factor through $R / I$, so also $\operatorname{Hom}_{R}(R / 2 I, R) \cong$ $R / I$. Finally, $\operatorname{Ext}_{R}^{1}(R / 2 I, R) \cong R /(2, I)$. One can see this from the exact 
sequence

$$
\begin{aligned}
0 & \rightarrow \operatorname{Hom}_{R}(R / 2 I, R) \cong R / I \cong J \rightarrow R \cong \operatorname{Hom}_{R}(R, R) \\
& \rightarrow \operatorname{Hom}_{R}(2 I, R) \cong I \rightarrow \operatorname{Ext}_{R}^{1}(R / 2 I, R) \rightarrow \operatorname{Ext}_{R}^{1}(R, R)=0 .
\end{aligned}
$$

So for example we have a short exact sequence

$$
\begin{aligned}
0 & \rightarrow \operatorname{Ext}_{R}^{1}\left(K_{G}^{0}\left(S^{3}\right), R\right) \cong R /(2, I) \rightarrow K_{1}^{G}\left(S^{3}\right) \cong R / 2 I \\
& \rightarrow \operatorname{Hom}_{R}\left(K_{G}^{1}\left(S^{3}\right), R\right) \cong R / I \rightarrow 0,
\end{aligned}
$$

and the sequence does not split by Proposition A.7. This resolves a question left unanswered in [21].

\section{References}

[1] O. Aharony, B. Fiol, D. Kutasov and David A. Sahakyan, Little string theory and heterotic/type II duality, Nucl. Phys. B 679 (2004), 3-65, arXiv: hep-th/0310197.

[2] T. Asakawa, S. Sugimoto and S. Terashima, D-branes and $K K$ theory in type-I string theory, J. High Energy Phys. 05 (2002), 007, arXiv: hep-th/0202165.

[3] K. Becker, M. Becker and John H. Schwarz, String theory and MTheory: a modern introduction. Cambridge University Press, Cambridge, 2007.

[4] K. Behrndt, E. Bergshoeff and B. Janssen, Type II duality symmetries in six dimensions, Nucl. Phys. B 467(1-2) (1996), 100-126, arXiv:hep-th/9512152.

[5] O. Bergman, Tachyon condensation in unstable type-I D-brane systems, J. High Energy Phys. 11 (2000), 015, arXiv:hep-th/0009252.

[6] O. Bergman, Eric G. Gimon and P. Horava, Brane transfer operations and T-duality of non-BPS states, J. High Energy Phys. 04 (1999), 010, arXiv:hep-th/9902160.

[7] V. Braun and B. Stefanski, Jr. Orientifolds and K-theory. 2002, arXiv: hep-th/0206158.

[8] A. Dabholkar, Ten-dimensional heterotic string as a soliton, Phys. Lett. B 357(3) (1995), 307-312, arXiv:hep-th/9506160.

[9] J. Distler, Daniel S. Freed and Gregory W. Moore, Orientifold precis. arXiv:0906.0795, 2009, arXiv:0906.0795. 
[10] S. Echterhoff and O. Pfante, Equivariant K-theory of finite dimensional real vector spaces, Münster J. Math. 2 (2009), 65-94, arXiv:0903.1035.

[11] M. Frau, L. Gallot, A. Lerda and P. Strigazzi, Stable non-BPS Dbranes in type I string theory, Nucl. Phys. B 564(1-2) (2000) 60-85, arXiv:hep-th/9903123.

[12] H. García-Compeán, D-branes in orbifold singularities and equivariant K-theory, Nucl. Phys. B 557(3) (1999), 480-504, arXiv:hep-th/9812226.

[13] E. Gava, J. F. Morales, K. S. Narain and G. Thompson, Bound states of type I D-strings, Nucl. Phys. B 528(1-2) (1998), 95-108, arXiv: hep-th/9801128.

[14] S. Gukov, K-theory, reality, and orientifolds, Comm. Math. Phys. 210(3) (2000), 621-639, arXiv:hep-th/9901042.

[15] P. Hořava and E. Witten, Heterotic and type I string dynamics from eleven dimensions, Nucl. Phys. B 460(3) (1996), 506-524, arXiv:hep-th/9510209.

[16] C. M. Hull, String-string duality in ten dimensions, Phys. Lett. B 357(4) (1995), 545-551, arXiv:hep-th/9506194.

[17] C. M. Hull and P. K. Townsend, Unity of superstring dualities, Nucl. Phys. B 438(1-2) (1995), 109-137, arXiv:hep-th/9410167.

[18] Clifford V. Johnson and Robert C. Myers, Aspects of type IIB theory on asymptotically locally Euclidean spaces, Phys. Rev. D (3) 55(10) (1997), 6382-6393, arXiv:hep-th/9610140.

[19] E. Kiritsis, N. Obers and B. Pioline, Heterotic/type II triality and instantons on $K_{3}$. J. High Energy Phys. 1 (2000), 029, January, arXiv:hep-th/0001083.

[20] D. Kutasov, Orbifolds and solitons, Phys. Lett. B 383 (1996), 48-53, arXiv:hep-th/9512145.

[21] Ib. Madsen and J. Rosenberg, The universal coefficient theorem for equivariant $K$-theory of real and complex $C^{*}$-algebras, in 'Index theory of elliptic operators, foliations, and operator algebras' (New Orleans, LA/Indianapolis, IN, 1986), volume 70 of Contemp. Math., 145-173. Amer. Math. Soc., Providence, RI, 1988.

[22] J. Majumder and A. Sen, 'Blowing up' D-branes on nonsupersymmetric cycles. J. High Energy Phys. 09 (1999), 004, arXiv:hep-th/9906109.

[23] J. McCleary, A User's Guide to Spectral Sequences, volume 58 of Cambridge Studies in Advanced Mathematics. Cambridge University Press, Cambridge, 2nd edn., 2001. 
"ATMP-16-6-A1-MEN" — 2013/5/22 — 21:05 — page 1618 — \#28

[24] G. Moore, K-theory from a physical perspective, in 'Topology, geometry and quantum field theory', volume 308 of London Math. Soc. Lecture Note Ser., 194-234. Cambridge University Press, Cambridge, 2004, arXiv:hep-th/0304018.

[25] K. Olsen and Richard J. Szabo, Constructing D-branes from K-theory, Adv. Theor. Math. Phys. 3(4) (1999), 889-1025, arXiv: hep-th/9907140.

[26] Rui M.G. Reis, Richard J. Szabo and A. Valentino, KO-homology and type I string theory, Rev. Math. Phys. 21(9) (2009), 1091-1143, arXiv:hep-th/0610177.

[27] G. Segal, Equivariant K-theory, Inst. Hautes Études Sci. Publ. Math. 34 (1968), 129-151.

[28] G. Segal, The representation ring of a compact Lie group, Inst. Hautes Études Sci. Publ. Math. 34 (1968), 113-128

[29] A. Sen, An introduction to duality symmetries in string theory, in 'Unity from duality: gravity, gauge theory and strings' (Les Houches, 2001), NATO Adv. Study Inst., 241-322. EDP Sci., Les Ulis, 2003.

[30] A. Sen, String-string duality conjecture in six dimensions and charged solitonic strings, Nucl. Phys. B 450(1-2) (1995), 103-114, arXiv: hep-th/9504027.

[31] A. Sen, Duality and orbifolds, Nucl. Phys. B 474(2) (1996), 361-375.

[32] A. Sen, SO(32) spinors of type I and other solitons on brane-antibrane pair, J. High Energy Phys. 09 (1998), 023, arXiv:hep-th/9808141.

[33] A. Sen, Type I D-particle and its interaction, J. High Energy Phys. 10 (1998), 021, arXiv:hep-th/9809111.

[34] A. Sen, Duality symmetries in string theory, Curr. Sci. 77(12) (1999), 1635-1645. Available at http://www.ias.ac.in/currsci/ dec251999/articles20.htm.

[35] A. A. Tseytlin, Heterotic-type I superstring duality and lowenergy effective actions, Nucl. Phys. B 467(3) (1996), 383-395, arXiv: hep-th/9512081.

[36] E. Witten, String theory dynamics in various dimensions, Nucl. Phys. B 443(1-2) (1995), 85-126, arXiv: hep-th/9503124.

[37] E. Witten, D-branes and K-theory, J. High Energy Phys. 12 (1998), 019, arXiv:hep-th/9810188.

[38] E. Witten, Overview of K-theory applied to strings, Internat. J. Modern Phys. A 16(5) (2001), 693-706, arXiv:hep-th/0007175. Strings 2000. Proceedings of the International Superstrings Conference (Ann Arbor, MI). 